\title{
Comprehensive analysis of genomic mutation signature and tumor mutation burden for prognosis of intrahepatic cholangiocarcinoma
}

Rui Zhang, Qi Li, Jialu Fu, Zhechuan Jin, Jingbo Su, Jian Zhang, Chen Chen, Zhimin Geng* and Dong Zhang*(D)

\begin{abstract}
Background: Intrahepatic cholangiocarcinoma (iCCA) is a highly lethal malignancy of the biliary tract. Analysis of somatic mutational profiling can reveal new prognostic markers and actionable treatment targets. In this study, we explored the utility of genomic mutation signature and tumor mutation burden (TMB) in predicting prognosis in iCCA patients.

Methods: Whole-exome sequencing and corresponding clinical data were collected from the ICGC portal and cBioPortal database to detect the prognostic mutated genes and determine TMB values. To identify the hub prognostic mutant signature, we used Cox regression and Lasso feature selection. Mutation-related signature (MRS) was constructed using multivariate Cox regression. The predictive performances of MRS and TMB were assessed using Kaplan-Meier (KM) analysis and receiver operating characteristic (ROC). We performed a functional enrichment pathway analysis using gene set enrichment analysis (GSEA) for mutated genes. Based on the MRS, TMB, and the TNM stage, a nomogram was constructed to visualize prognosis in iCCA patients.

Results: The mutation landscape illustrated distributions of mutation frequencies and types in iCCA, and generated a list of most frequently mutated genes (such as Tp53, KRAS, ARID1A, and IDH1). Thirty-two mutated genes associated with overall survival (OS) were identified in iCCA patients. We obtained a six-gene signature using the Lasso and Cox method. AUCs for the MRS in the prediction of 1-, 3-, and 5-year OS were 0.759, 0.732, and 0.728, respectively. Kaplan-Meier analysis showed a significant difference in prognosis for patients with iCCA having a high and low MRS score $(P<0.001)$. GSEA was used to show that several signaling pathways, including MAPK, PI3KAKT, and proteoglycan, were involved in cancer. Conversely, survival analysis indicated that TMB was significantly associated with prognosis. GSEA indicated that samples with high MRS or TMB also showed an upregulated expression of pathways involved in tumor signaling and the immune response. Finally, the predictive nomogram (that included MRS, TMB, and the TNM stage) demonstrated satisfactory performance in predicting survival in patients with iCCA.

(Continued on next page)
\end{abstract}

\footnotetext{
*Correspondence: gengzhimin@mail.xjtu.edu.cn; zhangdong811021@126.com

Department of Hepatobiliary Surgery, The First Affiliated Hospital of Xi'an Jiaotong University, Xi'an 710061, China
}

C C The Author(s). 2021 Open Access This article is licensed under a Creative Commons Attribution 4.0 International License, which permits use, sharing, adaptation, distribution and reproduction in any medium or format, as long as you give appropriate credit to the original author(s) and the source, provide a link to the Creative Commons licence, and indicate if changes were made. The images or other third party material in this article are included in the article's Creative Commons licence, unless indicated otherwise in a credit line to the material. If material is not included in the article's Creative Commons licence and your intended use is not permitted by statutory regulation or exceeds the permitted use, you will need to obtain permission directly from the copyright holder. To view a copy of this licence, visit http://creativecommons.org/licenses/by/4.0/. The Creative Commons Public Domain Dedication waiver (http://creativecommons.org/publicdomain/zero/1.0/) applies to the data made available in this article, unless otherwise stated in a credit line to the data. 
(Continued from previous page)

Conclusions: Mutation-related signature and TMB were associated with prognosis in patients with iCCA. Our study provides a valuable prognostic predictor for determining outcomes in patients with iCCA.

Keywords: Genomic mutation signature, Tumor mutation burden, Intrahepatic cholangiocarcinoma, Prognostic biomarker, Nomogram

\section{Background}

Cholangiocarcinoma (CCA) is a highly lethal and aggressive malignancy originating from the biliary epithelium. Based on the anatomical site of origin, CCA can be classified into three subtypes including perihilar (pCCA), distal (dCCA), and intrahepatic (iCCA) [1]. iCCA accounts for $10-20 \%$ of primary liver cancers and approximately $20 \%$ of biliary-tract cancers [2]. Postoperative 5year overall survival (OS) in iCCA patients is poor (30$40 \%)[3,4]$. In the last decade, iCCA has shown an increasing incidence rate and mortality worldwide, which contrasts with the decreasing trends shown by pCCA and dCCA [5]. Surgical resection is currently the mainstay of curative-intent treatment for patients in the early stage; however, the vast majority of patients miss the opportunity for radical surgery [6, 7]. Nevertheless, even after resection, iCCA patients show a high incidence of recurrence [8-10]. These findings highlight the importance of discovering novel prognostic biomarkers and constructing predictive models for patients with iCCA. Such approaches can be used to make treatment determinations and improve patient prognosis.

Cancer is often accompanied by an accumulation of various genetic mutations. Accumulated somatic mutations contribute to tumorigenesis and progression of malignancy. Genetic mutations are consistently present and critical factors that determine gene function and biological behavior in malignant tumors [11, 12]. Certain genetic mutations may be used as prognostic indicators to predict patient survival and response to adjunctive therapy [13-16]. Several frequently occurring genetic alterations, including TP53, KRAS, ARID1A, IDH1/2, $B A P 1$, and PBRM1, have been identified in ICCA [17, 18]. However, the prognostic implications of these somatic alterations in iCCA are poorly understood. Improving our understanding of these genetic mutations is vital for selecting prognostic genetic biomarkers, identifying high-risk CCA patients harboring pertinent genetic mutations, and tailoring treatment strategies in clinical practice.

Tumor mutation burden (TMB) is defined as the number of somatic (such as missense, deletion, or insertion) mutations per megabase of genome examined [19]. Recent studies have indicated that TMB may be used as a biomarker to predict patient response to immune checkpoint inhibitor (ICI) therapy [20, 21]. Furthermore, numerous studies have shown that TMB can be used to predict the effectiveness of immunotherapy against various cancers such as non-small cell lung carcinoma (NSCLC) [22, 23], melanoma [24], esophagogastric [25], and colorectal [26]. Although the utility of TMB in predicting the effectiveness of ICIs has been established, few studies have investigated the prognostic potential of TMB in predicting the survival of patients with iCCA.

Whole exome sequencing (WES) is regarded as the gold standard for assessing TMB values. In recent years, bioinformatic WES resources have become available from public databases, such as the International Cancer Genome Consortium (ICGC, https://dcc.icgc.org/) and cBioPortal (https://www.cbioportal.org/), enabling largescale genomic integration and comprehensive bioinformatics analysis of various cancers. These public databases can be used to determine factors that influence anti-cancer immunotherapy.

In this study, we used WES data from ICGC and cBioPortal database to investigate the mutational landscape of iCCA; explore the potential impact of mutationrelated signature on patient survival; and establish a reliable nomogram model based on mutant gene signature, $\mathrm{TMB}$, and other clinical characteristics to predict the OS of patients with iCCA. The findings obtained in our present study, as well as our nomogram model, can be used to explore new potentially prognostic biomarkers and provide therapeutic targets for the treatment of patients with iCCA.

\section{Methods \\ Collection of mutation data}

Data on somatic mutations and the corresponding clinicopathological characteristics of iCCA patients were acquired from cBioPortal (http://www.cbioportal.org) and the ICGC portal (http://dcc.icgc.org/releases/current/ Projects). We selected the WES dataset for iCCA patients only. The repositories used were BTCA-JP (Japan, Nat Genet 2015) [27], BTCA-SG (Singapore, Cancer Discov 2017) [2], TCGA-CHOL (TCGA, PanCancer Atlas) [12] and Intrahepatic Cholangiocarcinoma (Shanghai, Nat Commun 2014) [28]. Only patients from these datasets with complete clinicopathological information were included. Clinical characteristics included age, gender, TNM stage, survival status, and survival time. Perl scripts were then used to extract the somatic 
mutation data on iCCA. The "GenVisR" and "karyoploteR" functions in the $\mathrm{R}$ software package were used to visualize the genetic landscape. Comparison of these mutations with those listed in the Cancer Hotspot Mutation database (https://www.cancerhotspots.org/\#/home) provided information on whether these mutations are predicted to be putative driver mutations. We also screened the top mutations by comparing them with the data available in OnkoKB (https://www.oncokb.org/) to determine whether any of these mutations are known to be clinically associated. We then used the "corrplot" package to explore correlations between mutations that co-occurred or were exclusive of each other. In order to show differences in mutation across different ethnic groups, we also used the "yyplot," "ggplot2," and "ggord" package to perform the principal component analysis (PCA). Finally, a carton workflow was plotted to describe our data/pipeline (Fig. 1a).

\section{Construction of protein-protein interaction (PPI) network}

We selected the top 200 most frequently mutated genes to investigate the possible interactive relationships between these genes. These genes were inputted into the STRING database (https://stringdb.org/) to generate the PPI network; a confidence score $>0.7$ was designated as the cut-off criterion. We also modified the PPI network using Cytoscape. Then, mutated gene nodes with an edge of $>5$ were extracted as the most important targets.

\section{Functional enrichment pathway analysis}

The top 300 most frequently mutated genes were selected to perform an enrichment pathway analysis. The "org. Hs.eg.db," "ggplot2," "clusterProfiler," and "enrichplot" packages were utilized for Gene Ontology (GO) analysis and analysis of the Kyoto Gene and Genome Encyclopedia (KEGG) pathways; FDR $<0.05$ was considered to be statistically significant.

\section{Hub prognostic mutant genes and construction of the prognostic model}

Next, we used the univariate Cox regression analysis to screen the hub mutated genes for use in iCCA prognosis. We performed a dimensionality reduction analysis of survival-associated mutant genes using the least absolute shrinkage and selection operator (Lasso) regression, and "survival" and "glmnet" packages in R. Lasso sub-selects prognostic mutant genes by imposing a penalty proportional to the contraction of the regression coefficient. We then performed a multivariate Cox regression analysis to establish the mutation related signature (MRS), which was calculated using the following formula:

$$
\mathrm{MRS}=\sum_{i=1}^{n}\left(\beta_{i} * \mathrm{Mut}_{i}\right)
$$

where $\beta_{i}$ is the coefficient and Mut $_{i}$ represents the mutation status of genes (if the status is Mutation, $\mathrm{Mut}_{\mathrm{i}}=1$; if the statue is Wild, Mut $\mathrm{i}_{\mathrm{i}} 0$ ). Subsequently, the 318 iCCA patients were classified into low and high groups according to the median MRS. OS was estimated to compare survival between the two groups, with $P$ value $<0.05$ indicating a significant difference. Receiver operating characteristic (ROC) curves were generated to evaluate the performance of MRS in predicting the 1-, 3-, and 5-year survival. Forest plots were also used to show the hazard ratio (HR) of selected prognostic mutated genes using the "survminer" package.
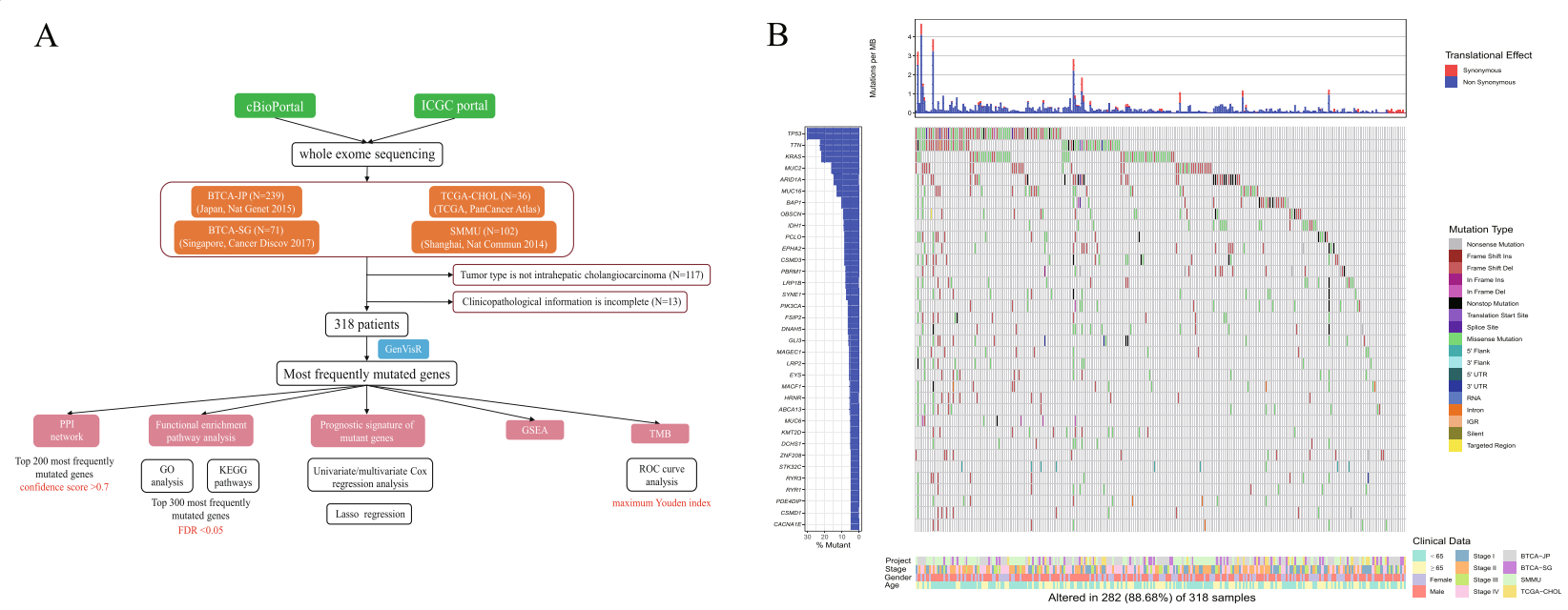

Fig. 1 Mutational landscape of iCCA. a Schematic view of patient cohorts and experimental pipeline used to analyze study data; $\mathbf{b}$ Top 35 frequently mutated genes are shown in the waterfall plot 
TMB values of patients with iCCA: estimation and prognostic analysis

TMB was defined as total number of mutations per coding area. All of the non-synonymous variants in the coding region were counted, and silent mutations were not considered. Genomic mutations for the 318 iCCA patients were specifically extracted. Because $38 \mathrm{Mb}$ is routinely used as total exon length in humans, we calculated TMB as total mutation frequency divided by 38 [29]. The TMB of each patient was calculated using this method, and corresponding survival data were merged. Then, we divided the patients into high- and low-TMB groups according to the cut-off TMB values, which were determined using the maximum Youden index of the ROC curve.

\section{Gene set enrichment analysis (GSEA)}

To examine the activity of potentially involved biologic pathways in the high- and low-group of patients based on MRS and TMB, we performed GSEA (MSigDB; version 7.1) using the "KEGG," "GO," and "immunologic signatures" gene sets from the Molecular Signature Database. The mutation gene list for the assessment of MRS and TMB status was used as input phenotype data. The analysis was performed 1000 times for gene-set permutations, and pathways with $P<0.05$ were considered statistically significant.

\section{Statistical analysis}

All statistical analyses were conducted using SPSS 24.0, $\mathrm{R}$ software (version 4.0.2), and GraphPad Prism 8.0. Student's t-test was used to compare continuous variables, and the $\chi^{2}$ test or Fisher's exact test was used to compare categorical data. The effects of AJCC TMN stage, MRS, and TMB on survival were assessed using the logrank test and Kaplan-Meier method. Multivariable Cox regression analysis was used to determine independent risk factors. Fisher's exact test was used to analyze the correlations between gene mutations that co-occurred or were exclusive of each other. A nomogram model was then constructed, and predictive performance of the nomogram was estimated using $\mathrm{C}$-index and calibration plot.

\section{Results}

\section{Landscape of genetic mutation profiles in iCCA}

The demographic and clinicopathological characteristics of 318 patients with iCCA, examined using WES, are listed in Table 1. Our cohort included 192 men and 126 women. Median age at the time of diagnosis was 62 years (range, 26-89 years). Our results revealed that 15 genes (TP53, TTN, KRAS, MUC2, ARID1A, MUC16, BAP1, OBSCN, CSMD3, EPHA2, IDH1, PCLO, LRP1B, PBRM1, and SYNE1) were mutated in
Table 1 Clinical characteristics of patients from ICGC and cBioPortal database

\begin{tabular}{|c|c|c|}
\hline \multirow[t]{2}{*}{ Variables } & \multicolumn{2}{|c|}{ All patients $(n=318)$} \\
\hline & Number (n) & Percent (\%) \\
\hline \multicolumn{3}{|l|}{ Age, years } \\
\hline Median & 62 & \\
\hline Range & $26-89$ & \\
\hline$<65$ & 178 & 56.0 \\
\hline$\geq 65$ & 140 & 44.0 \\
\hline \multicolumn{3}{|l|}{ Gender } \\
\hline Female & 126 & 39.6 \\
\hline Male & 192 & 60.4 \\
\hline \multicolumn{3}{|l|}{ TNM Stage } \\
\hline Stage I & 80 & 25.2 \\
\hline Stage II & 94 & 29.6 \\
\hline Stage III & 37 & 11.6 \\
\hline Stage IV & 107 & 33.6 \\
\hline \multicolumn{3}{|l|}{ Project } \\
\hline TCGA-CHOL & 32 & 10.0 \\
\hline BTCA-JP & 136 & 42.8 \\
\hline BTCA-SG & 48 & 15.1 \\
\hline SMMU & 102 & 32.1 \\
\hline \multicolumn{3}{|l|}{$\mathrm{TMB}$, mut/Mb } \\
\hline Median & 1.25 & \\
\hline Range & $0.03-54.74$ & \\
\hline$<10$ & 306 & 96.2 \\
\hline$\geq 10$ & 12 & 3.8 \\
\hline
\end{tabular}

more than 20 samples. The genetic mutation frequency is shown in the Supplementary Table 1. We visualized the landscape of mutation profiles using the "GenVisR" package, which shows only the top 35 most frequently mutated genes across the 318 samples (Fig. 1b). Moreover, we mapped the mutated genes whose mutation frequency was more than 5 on the chromosomes; in this map, the color red indicates the high-frequency mutation sites (Supplementary Fig. 1A). In addition, we provided a lollipop plot for the top five mutated genes (TP53, KRAS, MUC2, ARID1A, and $M U C 16)$ as Supplementary Fig. 1B. We also explored the correlation between mutations that cooccurred or were exclusive of each other (Supplementary Fig. 2A). Our results indicate that IDH1 was recurrent with BAP1/PBRM1/ARID1A/PIK3CA, but exclusive of KRAS/TP53/MUC2. We also conducted a PCA to examine our cohort of iCCA patients with respect to their different ethnic backgrounds. Our results indicate no difference in mutational pattern between different ethnic populations (Supplementary Fig. 2B). We compared the mutations occurring in 
our patient cohort with those listed in the Cancer Hotspot Mutation database, and found that 8 (TP53, KRAS, ARID1A, BAP1, IDH1, PBRM1, PIK3CA, and $K M T 2 D)$ of the top 35 mutations were predicted to be putative driver mutations. We also screened the top mutations to find actionable genes on the OnkoKB website. Our findings indicate that three genetic mutations (IDH1, KRAS, and PIK3CA) were potentially viable molecular alterations.

\section{PPI network of mutant genes}

The top 200 most frequently mutated genes were selected to construct a protein-protein interaction network (PPI). We performed a PPI network-based analysis using STRING database to determine whether the mutated genes functionally interacted with each other and were involved in tumorigenesis. The networks were also visualized using Cytoscape, which contained 108 nodes and 182 interacting pairs (Fig. 2a). The top hub genes with the highest clustering included TP53, PIK3CA, KRAS, NRAS, PTEN, $A N K 2$, SPTA1, ANK3, and ARID1A (Fig. 2b).
Functional pathway analysis of hub mutant signature We used the $\mathrm{R}$ software package to perform a functional pathway analysis of the top 300 mutant genes. Figure 2c shows the top 30 enriched GO terms associated with regulation of signaling in multicellular organisms, ion transmembrane transport, and transmembrane transporter complex and ion-gated channel activity. In addition, KEGG pathway analysis demonstrated enrichment of mutant genes in several signaling pathways involved in malignancy, such as the PI3K-AKT, MAPK, proteoglycan, and calciumsignaling pathways. We also expanded the pathway to include major genes from the enriched pathways (PI3K-AKT, MAPK, Cellular senescence and apoptosis pathway) (Fig. 2d).

\section{Prognostic signature of mutant genes}

To explore the prognostic roles of gene mutations in iCCA, we used univariate Cox regression to analyze patient survival. Patients were categorized into wildtype and mutant-type according to their genemutation status. Thirty-two mutated genes,

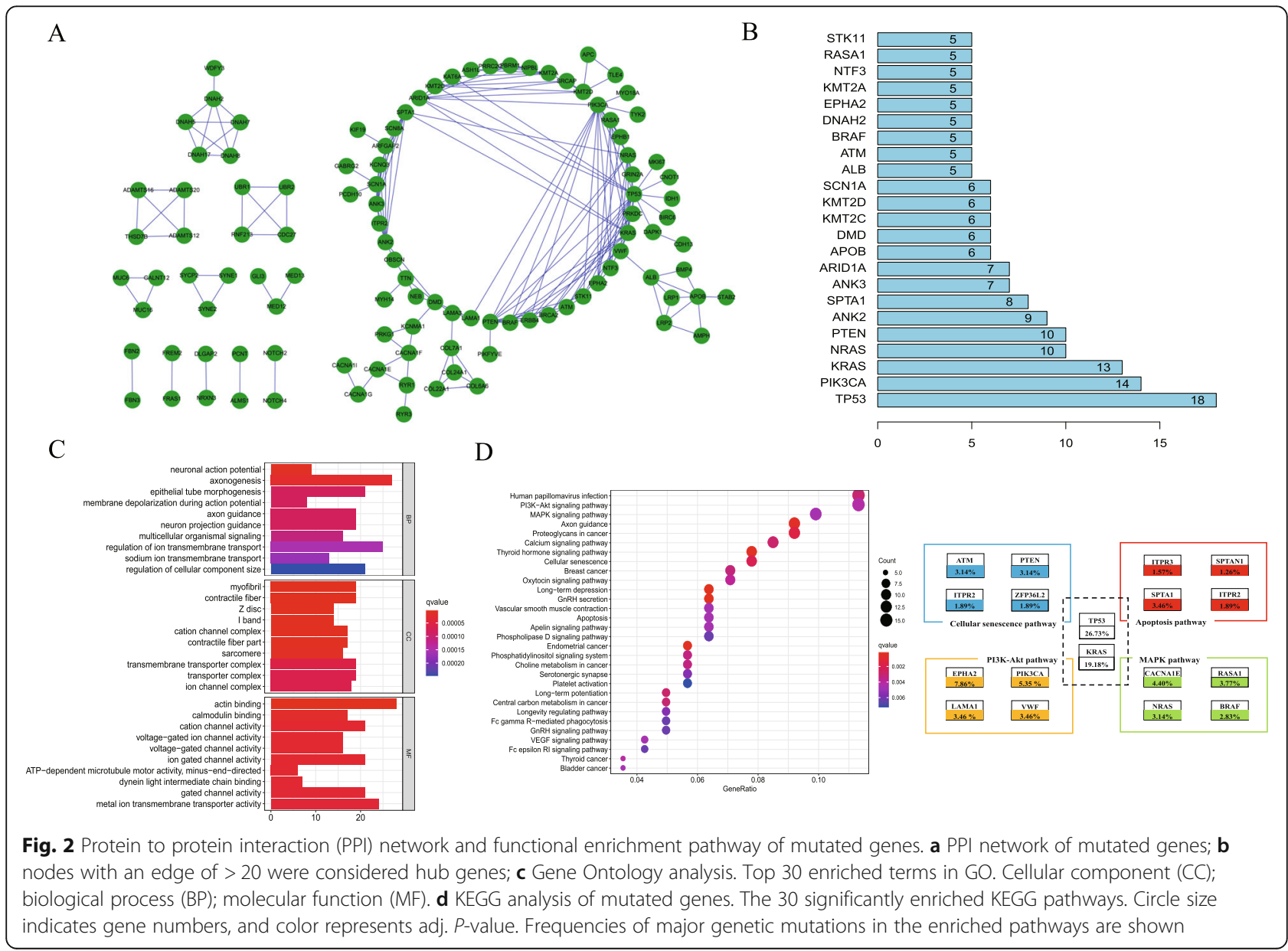


significantly associated with OS, were obtained, and the Kaplan-Meier analysis was used to assess their prognostic value (Fig. 3a, Supplementary Fig. 3 and 4). Furthermore, we obtained a gene signature for 12 prognostic mutated genes using the Lasso Cox method (Fig. 3b and c). We further utilized multivariate Cox regression analysis to establish a model that included six mutated genes to predict the survival of patients with iCCA (Fig. 3d). Using multivariate Cox regression analysis, regression coefficients were weighted for the six mutant genes to establish a risk- prediction model. MRS was calculated as follows: MRS $=(0.9772 \times$ CDC27 $)+(3.3262 \times$ AAK1 $)+(1.0356 \times$ TP53 $)+(0.8040 \times$ RBM10 $)+(0.5645 \times$ KRAS $)+(1.4581 \times$ IPO5) (Table 2). Based on the MRS value, patients were divided into the high- and low-risk groups. Patients in the low-risk group showed improved survival compared with that of patients in the high-risk group $(P<0.001$; Fig. $3 e)$. Our results indicate that using MRS to predict 1-, 3-, and 5 -year survival yielded an AUC value of 0.759, 0.732, and 0.728 , respectively (Fig. 3f), indicating high prediction efficiency.
A

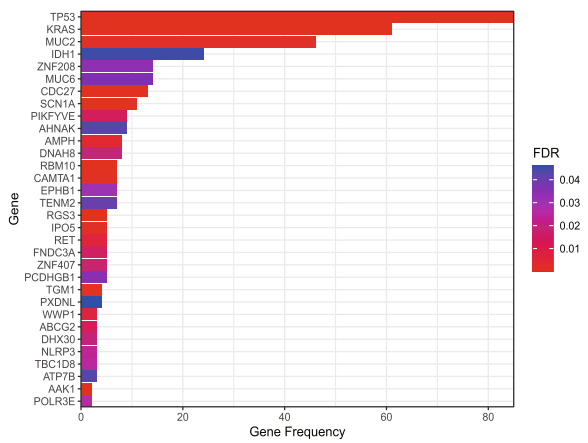

C

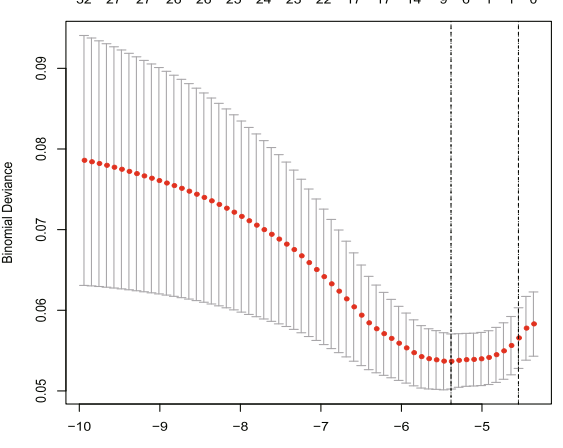

E

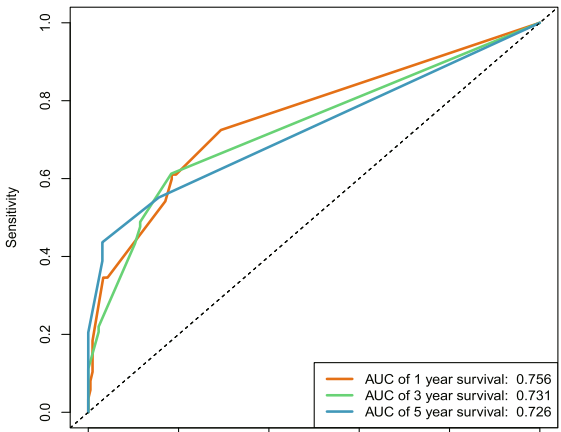

B

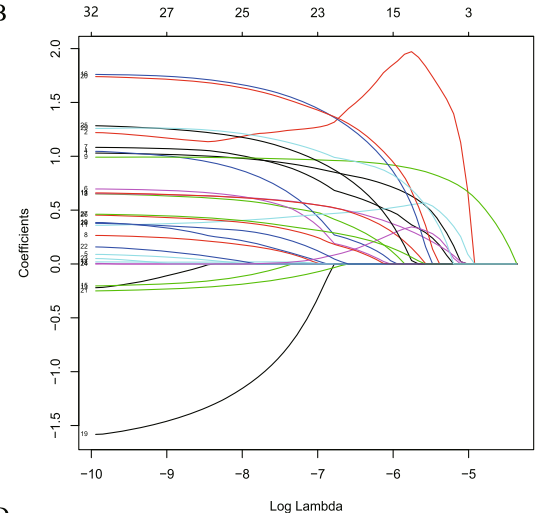

D

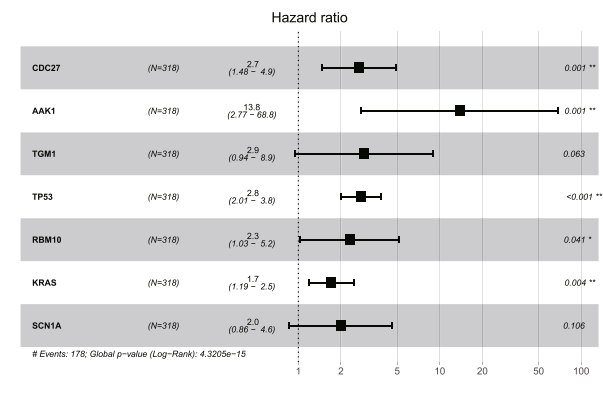

F

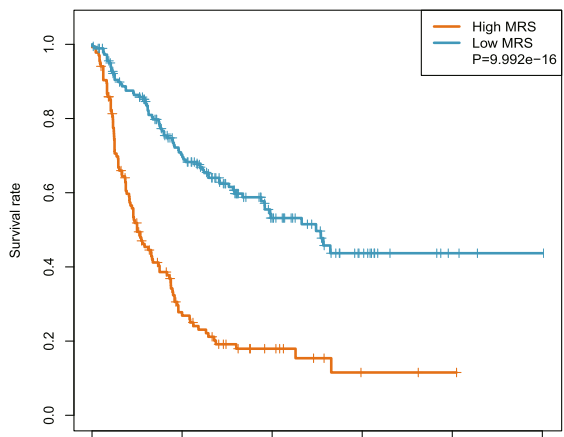

Fig. 3 Lasso regression was used to screen the MRS for the predictive model; $\mathbf{a}$ a bar plot shows 32 mutant genes significantly associated with OS; $\mathbf{b}$ Lasso coefficient profiles of mutated genes in our iCCA cohort. c A coefficient profile plot was generated to find the optimal parameter (lambda). d Hazard ratios (HR) for the selected prognostic mutated genes were generated using multivariate Cox analysis and are shown in Forest plots; e KM plotter shows differences between the high- and low-MRS groups, indicating that high MRS was associated with poor survival outcomes. f Time-dependent ROC curve analysis shows that the AUCs for MRS were 0.759, 0.732, and 0.728 for 1, 3, and 5-year OS, respectively; this analysis demonstrates the satisfactory predictive accuracy of the MRS model 
Table 2 6- mutation gene risk signature from multivariable Cox regression analysis

\begin{tabular}{lllll}
\hline Gene & Coefficient & HR & $\mathbf{9 5 \% ~ C l}$ & P-value \\
\hline CDC27 & 0.9772 & 2.657 & $1.463-4.826$ & 0.001333 \\
AAK1 & 3.3263 & 27.835 & $6.606-117.292$ & $5.83 \mathrm{e}-06$ \\
TP53 & 1.0356 & 2.817 & $2.050-3.871$ & $1.70 \mathrm{e}-10$ \\
RBM10 & 0.8040 & 2.235 & $1.001-4.987$ & 0.049659 \\
KRAS & 0.5645 & 1.759 & $1.227-2.520$ & 0.002095 \\
IPO5 & 1.4581 & 4.298 & $1.743-10.600$ & 0.001547
\end{tabular}

Abbreviations: $\mathrm{Cl}$ confidence interval; HR hazard ratio

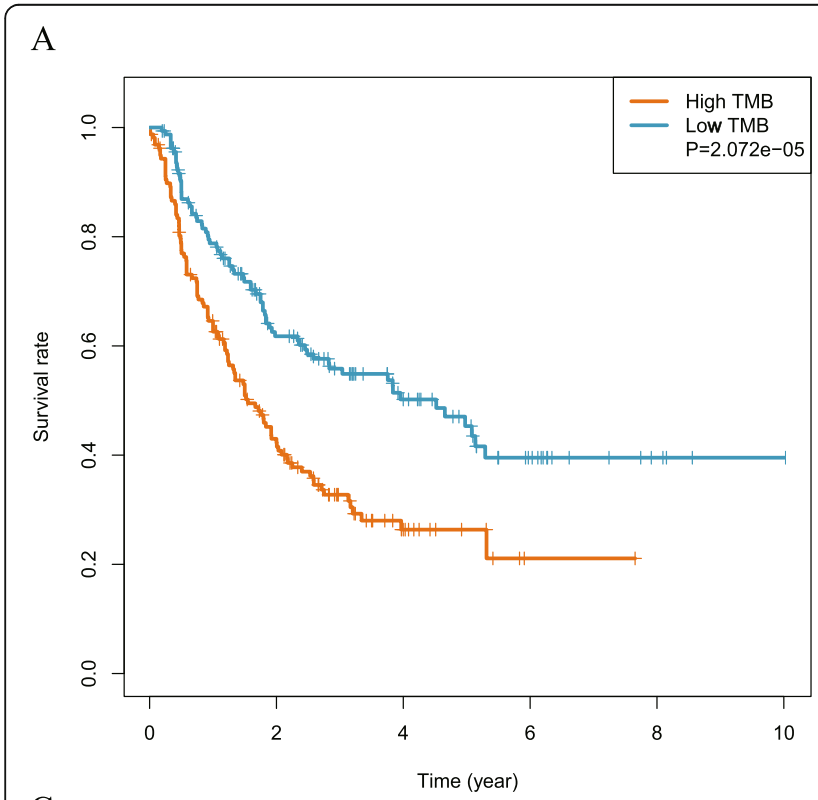

C

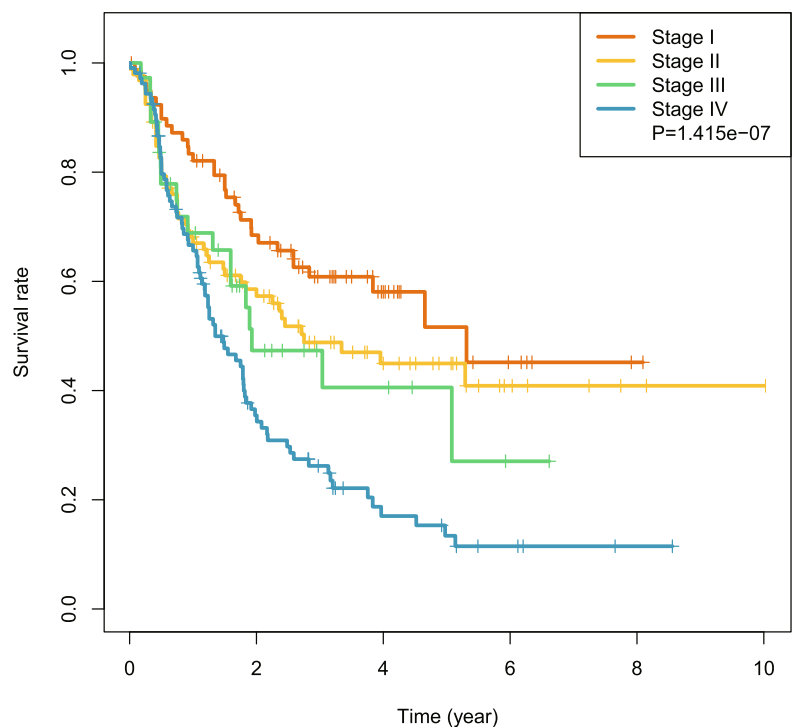

Prognostic impact of TMB and TNM stage in patients with iCCA

Next, we analyzed the utility of TMB in prognosis. Median TMB was 1.29 mutations/Mb (range, 0.03-54.74 mutations $/ \mathrm{Mb})$. We then analyzed the predictive performance of TMB with respect to OS. AUC of the ROC curve for TMB with respect to 1-, 3-, and 5-year survival was $0.776,0.685$, and 0.621 , respectively (Fig. $4 \mathrm{~b}$ ). TMB threshold values were calculated using the 3-year ROC curve analysis with maximum Youden index. We found

B

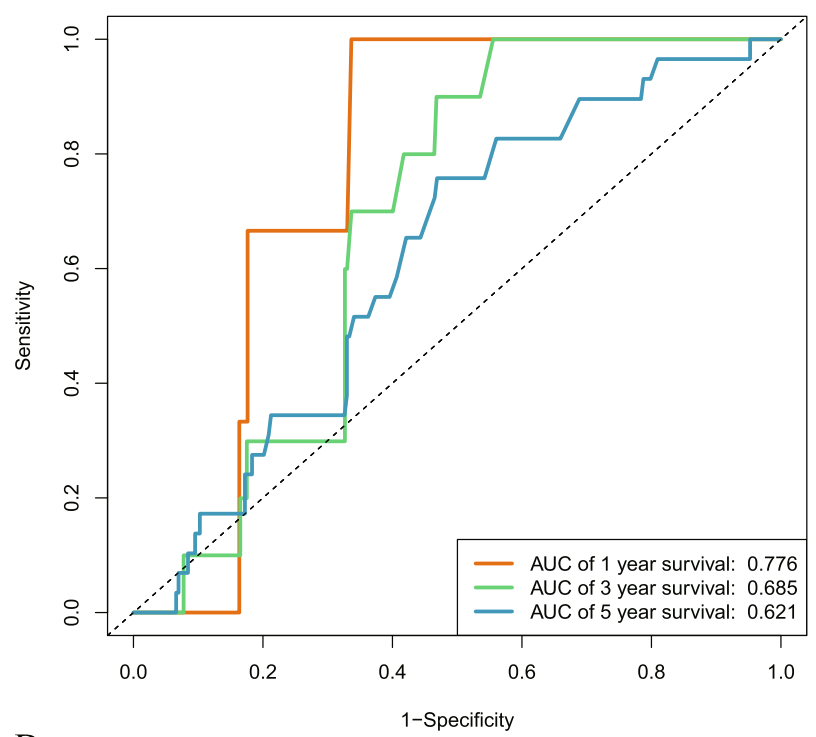

$\mathrm{D}$

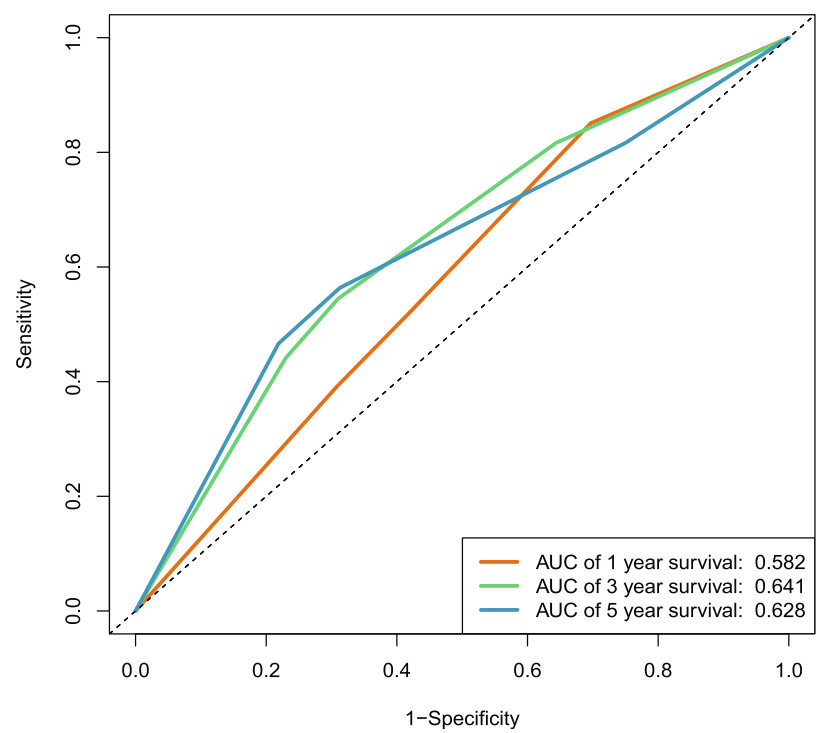

Fig. 4 Prognostic ability of TMB in predicting the OS of iCCA patients. a Kaplan-Meier plot, used to analyze the difference in survival of the highand low-TMB group, shows that the high-TMB group had poor survival outcomes. b Time-dependent ROC curve analysis shows that the AUCs for TMB were $0.776,0.685$, and 0.621 for 1,3 , and 5 -year OS, respectively; this analysis demonstrates the satisfactory predictive accuracy of the TMB model. c Kaplan-Meier plot shows significant differences between patients grouped with respect to their TNM stage. $\mathbf{d}$ Time-dependent ROC curve analysis of the TNM stage 
that maximal AUC value was achieved when the cut-off value of TMB was 1.29. Therefore, we defined $1.29 \mathrm{mu}-$ tations $/ \mathrm{Mb}$ as the cut-off value, classifying $\mathrm{TMB}>1.29$ mutations/Mb as the high group $(N=157)$ and $\mathrm{TMB} \leq$ 1.29 mutations/Mb as the low group $(N=161)$. KM plotter of survival analysis showed that OS was significantly decreased in patients with high TMB compared with those having low TMB $(P<0.001$; Fig. $4 a)$. We also explored the relationship between TMB and prognostic mutant genes. Our results indicate that TMB was

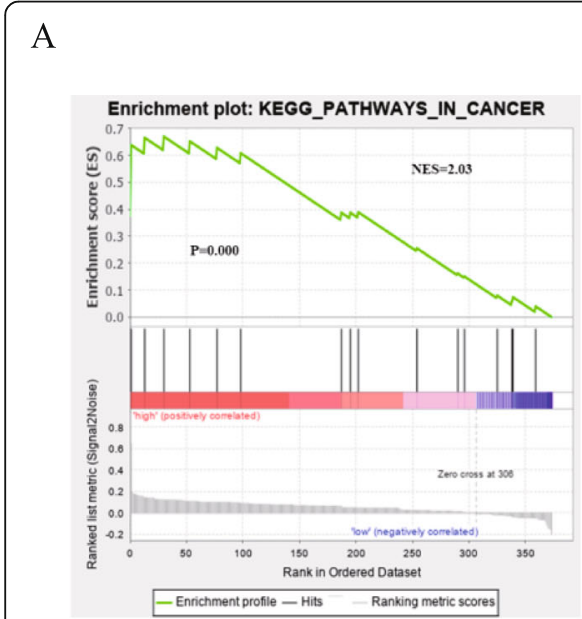

B
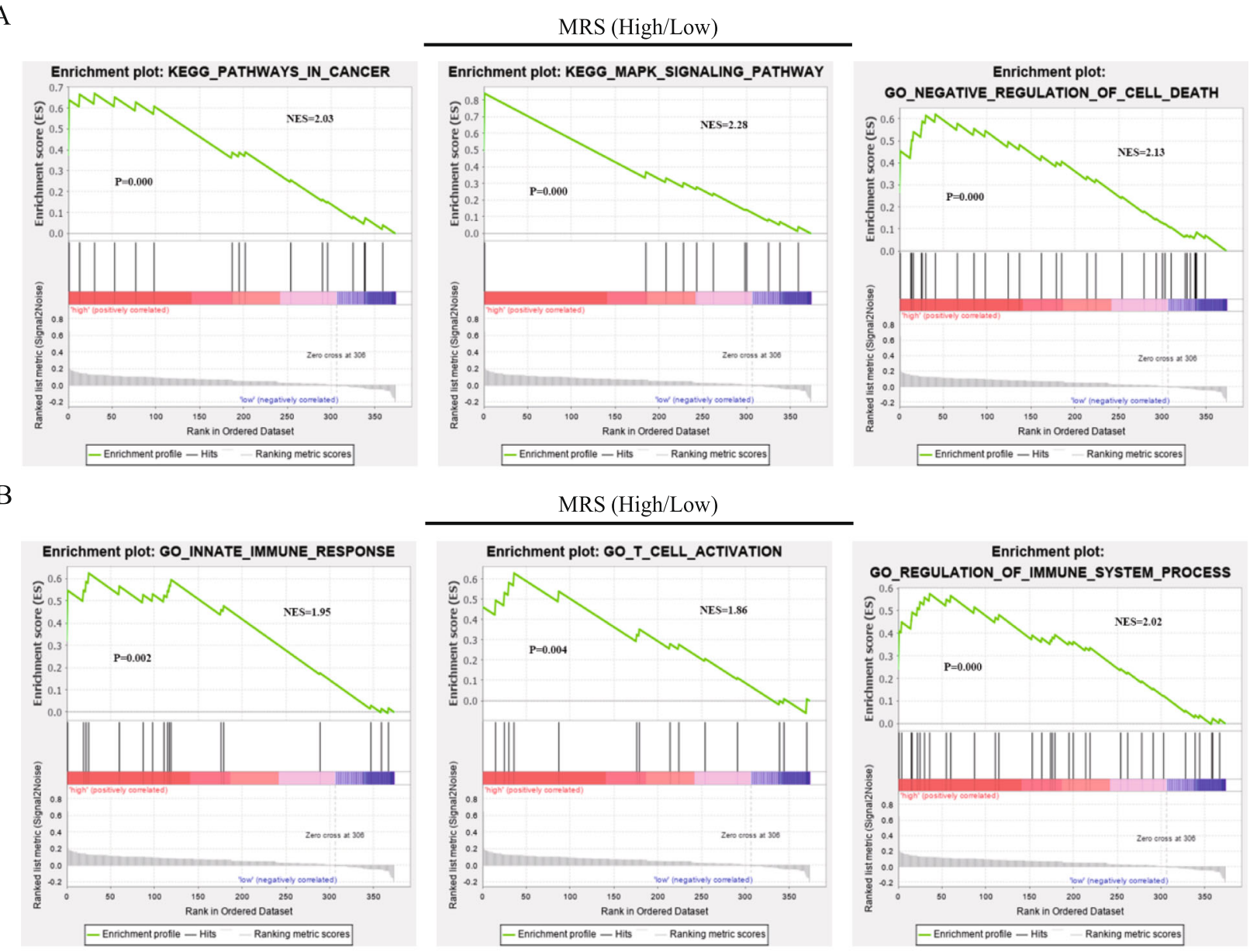

$\mathrm{C}$
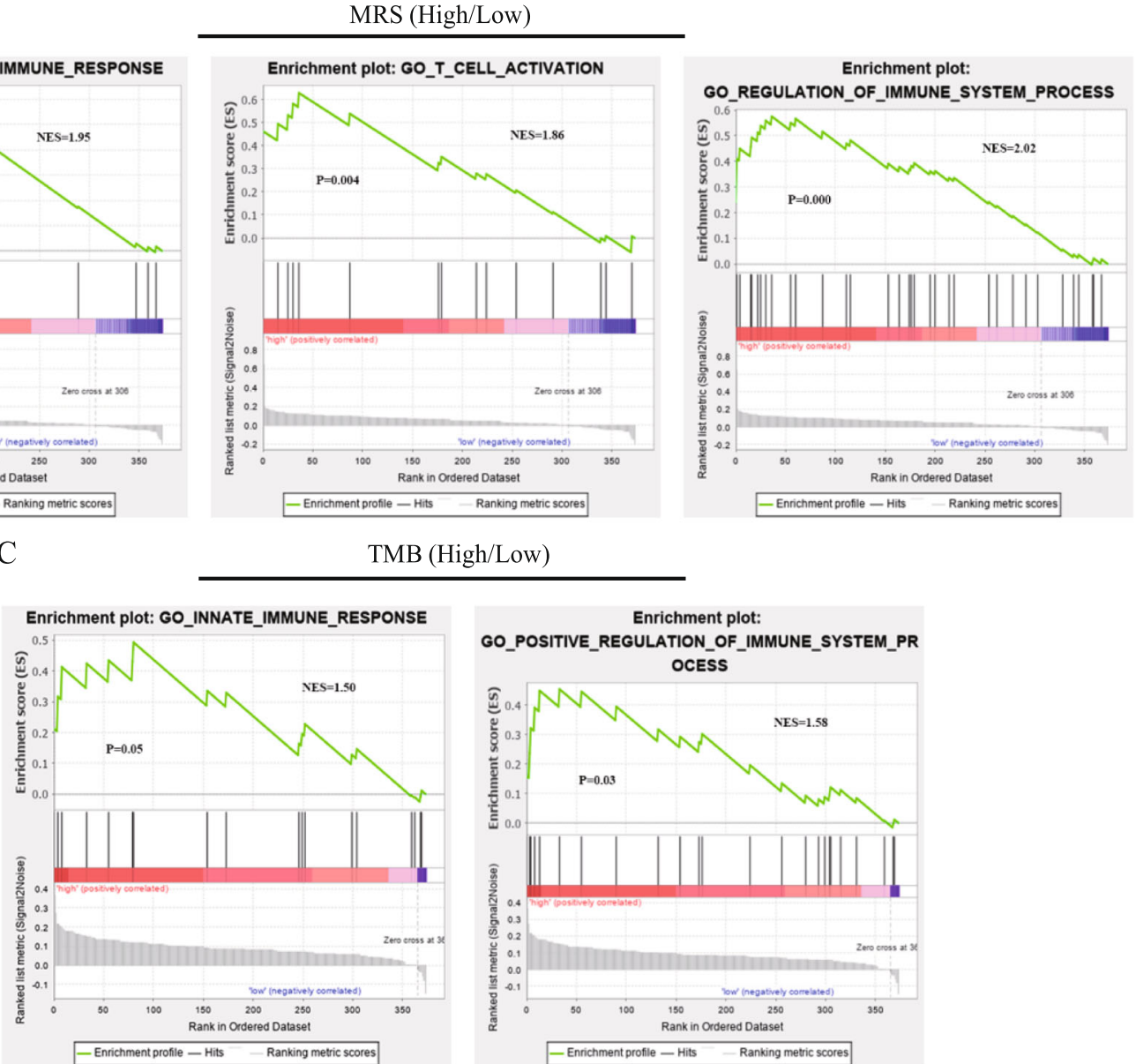

Fig. 5 Functional analysis of different MRS and TMB groups conducted using gene set enrichment analysis (GSEA). a and b Representative KEGG pathways and GO pathways analyzed in the low-MRS versus high-MRS group. $\mathbf{c}$ Representative KEGG pathways and GO pathways analyzed in the low-TMB versus high-TMB group 
moderately correlated with PIKFYVE $(r=0.31)$ and RGS3 ( $r=0.34)$ (Supplementary Fig. 5). In addition, we explored the prognostic role of TNM stage in iCCA. Our results demonstrate that TNM stage was significantly correlated with OS; however, AUC of the TNM stage in predicting 1-, 3-, and 5-year survival was 0.582 , 0.641 , and 0.628 , respectively, indicating a poor prognostic performance compared with those of MRS and TMB (Fig. 4c and d).

\section{Gene set enrichment analysis}

We used GSEA to visualize the enriched biological processes in the different MRS and TMB groups. Our results indicate that patients in the high MRS group were prone to show associations with the innate immune response, negative regulation of cell death, positive regulation of immune system processes, T-cell activation, MAPK signaling pathway, and cancer pathways (Fig. 5a and $b$ ). The high TMB group was enriched in positive regulation of immune-system processes and cancer pathways. These results demonstrate that crosstalk involved in tumor-signaling pathways and immune-system processes was upregulated in patients with high MRS or TMB (Fig. 5c).

\section{Construction and evaluation of the nomogram}

To find the independent prognostic biomarkers, we used univariate Cox regression analysis to analyze the associations between OS and factors such as MRS, age, sex, TNM stage, and TMB (Table 3). The results of multivariate Cox regression analysis demonstrated that TNM stage, MRS, and TMB were independent-risk predictors for iCCA (Table 3). We constructed a predictive nomogram based on these risk factors, which included MRS, TMB, and TNM stage (Fig. 6a). The C-index value for the nomogram was 0.721 (95\% CI, 0.613-0.829). Calibration curve indicated that the observed and predicted values were consistent in predicting OS (Fig. 6b).

\section{Discussion}

In this study, we explored the role of mutational signature and TMB in predicting the survival of patients with iCCA. First, whole exome sequencing (WES) data on iCCA were obtained from two public databases (ICGC and cBioportal), and frequently mutated genes were identified. Next, univariate, Lasso, and multivariate Cox regression analyses were used to screen for hub prognostic mutant signature and establish a mutation-risk model for predicting prognosis. After the prognostic role of MRS was confirmed, we used PPI, GO, KEGG, and GSEA analyses to reveal the potential cancer-related crosstalk involved. We also found that increased TMB was associated with poor prognosis. Furthermore, MRS, $\mathrm{TMB}$, and TNM stages were confirmed as independent
Table 3 Univariable and multivariable analysis of overall survival

\begin{tabular}{|c|c|c|c|c|c|c|}
\hline & \multicolumn{3}{|c|}{ Univariable } & \multicolumn{3}{|c|}{ Multivariable } \\
\hline & $\mathrm{HR}$ & $95 \% \mathrm{Cl}$ & P-value & $\mathrm{HR}$ & $95 \% \mathrm{Cl}$ & $P$-value \\
\hline \multicolumn{7}{|c|}{ Age, years } \\
\hline \multicolumn{7}{|l|}{$<65$} \\
\hline$\geq 65$ & 0.869 & $0.643-1.175$ & 0.362 & & & \\
\hline \multicolumn{7}{|l|}{ Gender } \\
\hline \multicolumn{7}{|c|}{ Female } \\
\hline Male & 0.686 & $0.787-1.439$ & 0.686 & & & \\
\hline \multicolumn{7}{|c|}{ TNM Stage } \\
\hline 1 & & & 0.000 & & & 0.000 \\
\hline$\|$ & 1.470 & $0.938-2.305$ & 0.093 & 1.717 & $1.092-2.698$ & 0.019 \\
\hline III & 1.743 & $0.987-3.079$ & 0.055 & 2.078 & $1.172-3.685$ & 0.012 \\
\hline IV & 2.716 & $1.799-4.101$ & 0.000 & 2.963 & $1.957-4.485$ & 0.000 \\
\hline \multicolumn{7}{|c|}{ TMB, mut/Mb } \\
\hline \multicolumn{7}{|l|}{ Low } \\
\hline High & 1.967 & $1.454-2.660$ & 0.000 & 1.500 & $1.085-2.073$ & 0.014 \\
\hline \multicolumn{7}{|l|}{ MRS } \\
\hline \multicolumn{7}{|l|}{ Low } \\
\hline High & 2.771 & $2.035-3.773$ & 0.000 & 2.448 & $1.758-3.409$ & 0.000 \\
\hline
\end{tabular}

Abbreviations: $\mathrm{Cl}$ confidence interval; $\mathrm{HR}$ hazard ratio

predictors for overall survival (OS) of patients with iCCA. Next, based on the risk factors affecting OS, we constructed a reliable nomogram model that demonstrated a satisfactory performance in predicting OS in patients with iCCA.

Gene mutations are ubiquitous in tumorigenesis and development of iCCA. Previous studies have reported comprehensive molecular alterations in biliary tract cancers $[18,28]$. In our study, the most relevant mutation was TP53 (26.7\%), followed by TTN (20.7\%), KRAS (19.1\%), MUC2 (14.5\%), and ARID1A (12.9\%), which was consistent with the findings of Cao et al. [30]. A study, based on approximately 500 patients with iCCA, used the three most recurrently mutated genes (IDH1, KRAS and TP53) to stratify patients into four subgroups, revealing distinct genomic and molecular features [31]. In our present study, we also found that several mutated genes, such as IDH1 (7.5\%), BAP1 (9.1\%), PBRM1 (7.2\%), and EPHA2 (7.9\%), were particular to iCCA. Jiao et al. performed exome sequencing on iCCA tissue samples, and found that frequently mutated genes (such as BAP1, ARID1A, and PBRM1) are involved in the chromatinremodeling pathway. Our results on the mutational frequency of chromatin-remodeling family genes in iCCA are consistent with those reported in previous studies [32]. We investigated the possible link between genetic alternations and patient prognosis, and found that the outcomes of patients with certain genetic mutations were worse than those of wild-type patients; statistically, 


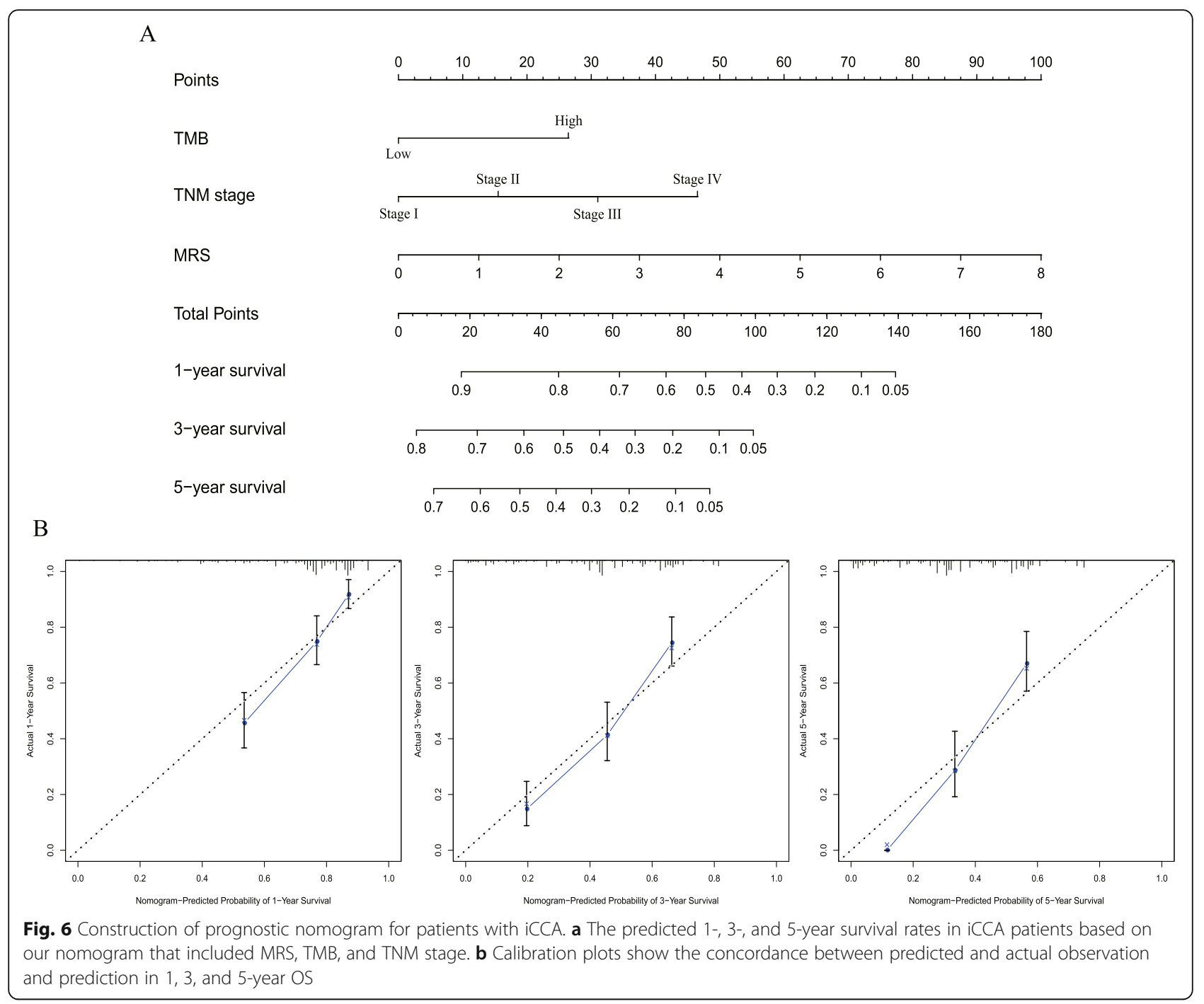

this finding showed borderline significance. Based on these prognostic factors, we developed a mutation risk score to predict survival. The MRS of our model was calculated based on 6 hub prognostic mutant genes (CDC27, AAK1, TP53, RBM10, KRAS, and IPO5). This MRS model showed high predictive accuracy for OS, was a reliable tool in predicting prognosis, and can be used in clinical practice. We next performed a functional pathway and GSEA analyses to uncover the molecular mechanisms underlying iCCA. Our functional enrichment pathway analysis indicated that the prognostic mutated genes were closely correlated with cancerassociated signaling pathways, such as cancer development and immune related pathway. GSEA also showed that the high MRS group was enriched in signaling involved in immune-related pathways.

In recent years, various types of immune checkpoint inhibitor (ICI) therapy have been developed for the treatment of patients with advanced-stage cancers.
However, only a minority of patients benefit from ICI therapies. TMB, a novel predictive biomarker, can predict a clinical response to ICI and can be used to identify patients likely to benefit from these therapies [19]. Increased TMB indicates increased levels of tumor antigen, which is beneficial for activating the body's immune response. Previous studies on TMB mostly focused on its capacity to predict the effectiveness of ICIs, showing a robust correlation between increased TMB and improved response to ICI therapy. However, few studies have explored the prognostic value of TMB in predicting the survival of patients with iCCA. Numerous studies have shown a relationship between TMB and survival in patients with cancer. Owada-Ozaki et al. found that increased TMB is correlated with decreased disease-free survival in NSCLC patients [33]. A study from China demonstrated that in HCC patients who had undergone radical resection, patients with increased TMB tend to 
show increased risk for recurrence; additionally, they also showed that TMB is an independent risk factor for RFS [34]. We show that the median TMB value in our iCCA patients was 1.25 (range 0.03-54.74). A large-scale examination of TMB in iCCA patients was performed by Cao et al. [30]. They used comprehensive genomic profiling to analyze the frequency and type of genetic aberrations, and did not observe genomic heterogeneity between Asian and Caucasian patients with iCCA; however, the relationship between TMB and prognosis was not evaluated in that study. Tian et al. investigated the genomic features of Chinese patients with iCCA, and explored the relationship between $\mathrm{TMB}$ and certain genetic changes [35]. It should be noted that the TMB of their cohort was greater than that determined in our study. This occurred because we only counted the non-synonymous variants.

In agreement with previous findings on other tumor types, our results show that increased TMB was correlated with poor patient prognosis. Therefore, we concluded that TMB had divergent prognostic impact in different patients with iCCA. In addition, our results indicate that a prognostic model incorporating TMB will likely improve prognostication and risk stratification in patients with iCCA.

In this study, we explored the prognostic role of MRS and TMB in patients with iCCA. Our findings indicate that the prognostic performance of the predictive model incorporating TMB or MRS was better than that of the TNM stage. Furthermore, results of our multivariate analysis indicate that TMB, MRS, and TNM stage were independent prognostic factors in iCCA. Despite these novel findings on the prognostic value of MRS and TMB, our study had several limitations. First, mutation data on iCCA were extracted from public databases that only included samples that had undergone WES. Targeted sequencing data were not used in our study. Additional WES data from patients with iCCA should be incorporated to reduce selective bias. Second, the mechanisms underlying the prognostic capability of MRS and TMB in iCCA should be further investigated. Additional experiments, performed both in vitro and in vivo, are required to support the results obtained in our present study. Finally, this study did not show whether specific mutations led to abnormal gene expression, and this question requires further investigation.

\section{Conclusion}

In summary, our study demonstrates that mutational signature and TMB were associated with prognosis in patients with iCCA. We visualized the mutational landscape and summarized the most commonly mutated genes. We also developed a risk model based on the prognostic utility of mutated genes, and found that MRS and TMB, included in the model, had divergent prognostic impacts in patients with iCCA. Based on independent risk factors, such as TNM stage, MRS, and TMB, we then constructed a reliable nomogram model for predicting OS in iCCA patients. The nomogram developed in this study can be incorporated into the methodology used for prognostication in patients with iCCA.

\section{Supplementary Information}

The online version contains supplementary material available at https://doi. org/10.1186/s12885-021-07788-7.

Additional file 1 Figure $\mathbf{S 1}$ The specific locations and types of gene mutations on iCCA patients. (a) Map shows specific locations of mutations on chromosomes. Green color indicates low-frequency mutation sites, while red represents high frequency; (b) lollipop plot of top five mutated genes.

Additional file $\mathbf{2}$ Figure $\mathbf{S 2}$ Correlation of gene mutations and PCA of iCCA patients based on different ethnic backgrounds. (a) Correlation of gene mutations that co-occurred or were exclusive of each other. (b) PCA of iCCA patients based on different ethnic backgrounds.

Additional file $\mathbf{3}$ Figure S3 and Figure S4 Kaplan-Meier plots of the 32 mutant genes significantly associated with OS.

Additional file 4 Figure S5 Relationship between TMB and prognostic mutant genes.

Additional file $\mathbf{5}$.

\section{Abbreviations}

iCCA: Intrahepatic cholangiocarcinoma; TMB: Tumor mutation burden; MRS: Mutation related signature; ICl: Immune checkpoint inhibitor; WES: Whole exome sequencing; ICGC: International Cancer Genome Consortium; GO: Gene Ontology; KEGG: Kyoto Gene and Genome Encyclopedia; Lasso: Least absolute shrinkage and selection operator regression; GSEA: Gene set enrichment analysis; PPI: Protein-protein interaction; HR: Hazard ratio; BP: Biological process; MF: Molecular function; CC: Cellular component; KM: Kaplan-Meier; ROC: Receiver operating characteristic; AUC: Area under the curve; CDC27: Cell division cycle 27; AAK1: AP2 associated kinase 1; TP53: Tumor protein P53; RBM10: RNA binding motif protein 10; IPO5: Importin 5; HCC: Hepatocellular carcinoma; NSCLC: Non-small cell lung carcinoma; AJCC: American Joint Committee on Cancer

\section{Acknowledgements}

We thank LetPub (www.letpub.com) for its linguistic assistance during the preparation of this manuscript.

\section{Authors' contributions}

$\mathrm{DZ}, \mathrm{ZG}$, and RZ designed the study; RZ, QL, ZJ, JS, and JZ analyzed the WES sequencing data, and performed bioinformatic and statistical analyses; RZ, JF and CC interpreted the data and drafted the manuscript; DZ and ZG revised the manuscript. All authors read and approved the final version of the manuscript.

\section{Funding}

This work was supported by the National Natural Science Foundation of China, No. 62076194, the Key Research and Development Plan of Shaanxi Province (No. 2017ZDXM-SF-055, No. 2017KW-060), the General Project of Natural Science Basic Research Plan of Shaanxi Province (No. 2020SF-070), and Clinical Research Fund of the First Affiliated Hospital of Xi'an Jiaotong University (XJTU1AF-CRF-2018-022). These funds were used for design of the 
study, collection and interpretation of data, bioinformatic analysis, and writing of the manuscript.

\section{Availability of data and materials}

All WES data and clinical information used in this study were acquired from the ICGC portal (http://dcc.icgc.org/releases/current/Projects) (up to June 10, 2019) and cBioPortal (http://www.cbioportal.org) (up to June 10, 2019).

\section{Ethics approval and consent to participate}

No ethics approval was required for the present study. The datasets analyzed in the present study are publicly available.

\section{Consent for publication}

Not applicable.

\section{Competing interests}

The authors declare that they have no competing interests.

Received: 3 October 2020 Accepted: 4 January 2021

Published online: 03 February 2021

\section{References}

1. Banales JM, Marin JJG, Lamarca A, Rodrigues PM, Khan SA, Roberts LR, Cardinale V, Carpino G, Andersen JB, Braconi C, et al. Cholangiocarcinoma 2020: the next horizon in mechanisms and management. Nat Rev Gastroenterol Hepatol. 2020;17(9):557-88.

2. Jusakul A, Cutcutache I, Yong CH, Lim JQ, Huang MN, Padmanabhan N, Nellore V, Kongpetch S, Ng AWT, Ng LM, et al. Whole-genome and Epigenomic landscapes of etiologically distinct subtypes of Cholangiocarcinoma. Cancer Discov. 2017;7(10):1116-35.

3. Mavros MN, Economopoulos KP, Alexiou VG, Pawlik TM. Treatment and prognosis for patients with intrahepatic Cholangiocarcinoma: systematic review and meta-analysis. JAMA Surg. 2014;149(6):565-74.

4. Buettner S, Galjart B, van Vugt JLA, Bagante F, Alexandrescu S, Marques HP, Lamelas J, Aldrighetti L, Gamblin TC, Maithel SK, et al. Performance of prognostic scores and staging systems in predicting long-term survival outcomes after surgery for intrahepatic cholangiocarcinoma. J Surg Oncol. 2017;116(8):1085-95.

5. Zhang H, Yang T, Wu M, Shen F. Intrahepatic cholangiocarcinoma: epidemiology, risk factors, diagnosis and surgical management. Cancer Lett. 2016;379(2):198-205.

6. Weber SM, Ribero D, O'Reilly EM, Kokudo N, Miyazaki M, Pawlik TM. Intrahepatic cholangiocarcinoma: expert consensus statement. HPB (Oxford). 2015;17(8):669-80.

7. Rizvi S, Khan SA, Hallemeier CL, Kelley RK, Gores GJ. Cholangiocarcinoma evolving concepts and therapeutic strategies. Nat Rev Clin Oncol. 2018; 15(2):95-111.

8. Chan KM, Tsai CY, Yeh CN, Yeh TS, Lee WC, Jan YY, Chen MF. Characterization of intrahepatic cholangiocarcinoma after curative resection: outcome, prognostic factor, and recurrence. BMC Gastroenterol. 2018;18(1):180.

9. Spolverato G, Kim Y, Alexandrescu S, Marques HP, Lamelas J, Aldrighetti L, Clark Gamblin T, Maithel SK, Pulitano C, Bauer TW, et al. Management and outcomes of patients with recurrent intrahepatic Cholangiocarcinoma following previous curative-intent surgical resection. Ann Surg Oncol. 2016; 23(1):235-43.

10. Zhang XF, Beal EW, Bagante F, Chakedis J, Weiss M, Popescu I, Marques HP, Aldrighetti L, Maithel SK, Pulitano C, et al. Early versus late recurrence of intrahepatic cholangiocarcinoma after resection with curative intent. $\mathrm{Br}$ J Surg. 2018;105(7):848-56.

11. Croce CM. Oncogenes and cancer. N Engl J Med. 2008;358(5):502-11.

12. Ding L, Bailey MH, Porta-Pardo E, Thorsson V, Colaprico A, Bertrand D, Gibbs DL, Weerasinghe A, Huang KL, Tokheim C, et al. Perspective on Oncogenic Processes at the End of the Beginning of Cancer Genomics. Cell. 2018; 173(2):305-20 e310.

13. Zhang C, Zheng Y, Li X, Hu X, Qi F, Luo J. Genome-wide mutation profiling and related risk signature for prognosis of papillary renal cell carcinoma. Ann Transl Med. 2019;7(18):427.

14. Smith JC, Sheltzer JM. Systematic identification of mutations and copy number alterations associated with cancer patient prognosis. eLife. 2018;7.
15. Buscail $L$, Bournet $B$, Cordelier P. Role of oncogenic KRAS in the diagnosis, prognosis and treatment of pancreatic cancer. Nat Rev Gastroenterol Hepatol. 2020;17(3):153-68.

16. Chae H, Kim D, Yoo C, Kim KP, Jeong JH, Chang HM, Lee SS, Park DH, Song TJ, Hwang $S$, et al. Therapeutic relevance of targeted sequencing in management of patients with advanced biliary tract cancer: DNA damage repair gene mutations as a predictive biomarker. Eur J Cancer. 2019;120:31-9.

17. Ma B, Meng H, Tian Y, Wang Y, Song T, Zhang T, Wu Q, Cui Y, Li H, Zhang $W$, et al. Distinct clinical and prognostic implication of IDH1/2 mutation and other most frequent mutations in large duct and small duct subtypes of intrahepatic cholangiocarcinoma. BMC Cancer. 2020; 20(1):318

18. Weinberg BA, Xiu J, Lindberg MR, Shields AF, Hwang JJ, Poorman $K$, Salem ME, Pishvaian MJ, Holcombe RF, Marshall $J$, et al. Molecular profiling of biliary cancers reveals distinct molecular alterations and potential therapeutic targets. J Gastrointest Oncol. 2019;10(4):652-62.

19. Samstein RM, Lee $C H$, Shoushtari AN, Hellmann MD, Shen R, Janjigian $Y Y$, Barron DA, Zehir A, Jordan EJ, Omuro A, et al. Tumor mutational load predicts survival after immunotherapy across multiple cancer types. Nat Genet. 2019;51(2):202-6.

20. Chan TA, Yarchoan M, Jaffee E, Swanton C, Quezada SA, Stenzinger A Peters S. Development of tumor mutation burden as an immunotherapy biomarker: utility for the oncology clinic. Ann Oncol. 2019;30(1):44-56.

21. Steuer CE, Ramalingam SS. Tumor mutation burden: leading immunotherapy to the era of precision medicine? J Clin Oncol. 2018;36(7): $631-2$.

22. Wang Z, Duan J, Cai S, Han M, Dong H, Zhao J, Zhu B, Wang S, Zhuo M, Sun J, et al. Assessment of blood tumor mutational burden as a potential biomarker for immunotherapy in patients with non-small cell lung Cancer with use of a next-generation sequencing Cancer gene panel. JAMA Oncol. 2019;5(5):696-702

23. Felip E, Navarro A, Callejo A, Marti AM, Cedres S, Pardo N, Ros J, Assaf JD, Pedrola A, Viaplana C, et al. Whole exome sequencing (WES) of non-small cell lung cancer (NSCLC) for tumor mutational burden (TMB) analysis and long-term benefit to immune checkpoint inhibitors (ICls). J Clin Oncol. 2019; 37:9071-1.

24. Dummer R, Brase JC, Garrett J, Campbell CD, Gasal E, Squires M, Gusenleitner D, Santinami M, Atkinson V, Mandalà M, et al. Adjuvant dabrafenib plus trametinib versus placebo in patients with resected, BRAF-mutant, stage III melanoma (COMBI-AD): exploratory biomarker analyses from a randomised, phase 3 trial. Lancet Oncol. 2020;21(3): 358-72

25. Greally M, Chou JF, Chatila WK, Margolis M, Capanu M, Hechtman JF, Tuvy Y, Kundra R, Daian F, Ladanyi M, et al. Clinical and molecular predictors of response to immune checkpoint inhibitors in patients with advanced Esophagogastric Cancer. Clin Cancer Res. 2019;25(20): 6160-9.

26. Schrock AB, Ouyang C, Sandhu J, Sokol E, Jin D, Ross JS, Miller VA, Lim D, Amanam I, Chao J, et al. Tumor mutational burden is predictive of response to immune checkpoint inhibitors in MSI-high metastatic colorectal cancer. Ann Oncol. 2019;30(7):1096-103.

27. Nakamura H, Arai Y, Totoki Y, Shirota T, Elzawahry A, Kato M, Hama N, Hosoda F, Urushidate T, Ohashi S, et al. Genomic spectra of biliary tract cancer. Nat Genet. 2015;47(9):1003-10.

28. Zou S, Li J, Zhou H, Frech C, Jiang X, Chu JS, Zhao X, Li Y, Li Q, Wang H, et al. Mutational landscape of intrahepatic cholangiocarcinoma. Nat Commun. 2014:5:5696

29. Chalmers ZR, Connelly CF, Fabrizio D, Gay L, Ali SM, Ennis R, Schrock A Campbell B, Shlien A, Chmielecki J, et al. Analysis of 100,000 human cancer genomes reveals the landscape of tumor mutational burden. Genome Med. 2017;9(1):34.

30. Cao J, Hu J, Liu S, Meric-Bernstam F, Zhao H. Intrahepatic Cholangiocarcinoma: Genomic Heterogeneity Between Eastern and Western Patients. JCO Precis Oncol. 2020;4(PO.18):00414. https://doi.org/10.1200/PO. 18.00414 PMID: 32923885; PMCID: PMC7446410.

31. Nepal C, O'Rourke CJ, Oliveira D, Taranta A, Shema S, Gautam P, Calderaro J, Barbour A, Raggi C, Wennerberg K, et al. Genomic perturbations reveal distinct regulatory networks in intrahepatic cholangiocarcinoma. Hepatology (Baltimore, Md). 2018;68(3):949-63. 
32. Jiao Y, Pawlik TM, Anders RA, Selaru FM, Streppel MM, Lucas DJ, Niknafs N, Guthrie VB, Maitra A, Argani P, et al. Exome sequencing identifies frequent inactivating mutations in BAP1, ARID1A and PBRM1 in intrahepatic cholangiocarcinomas. Nat Genet. 2013;45(12): 1470-3.

33. Owada-Ozaki Y, Muto S, Takagi H, Inoue T, Watanabe Y, Fukuhara M, Yamaura T, Okabe N, Matsumura Y, Hasegawa T, et al. Prognostic impact of tumor mutation burden in patients with completely resected non-small cell lung Cancer: brief report. J Thorac Oncol. 2018;13(8):1217-21.

34. Cai H, Zhang Y, Zhang H, Cui C, Li C, Lu S. Prognostic role of tumor mutation burden in hepatocellular carcinoma after radical hepatectomy. J Surg Oncol. 2020;121(6):1007-14.

35. Tian W, Hu W, Shi X, Liu P, Ma X, Zhao W, Qu L, Zhang S, Shi W, Liu A, et al. Comprehensive genomic profile of cholangiocarcinomas in China. Oncol Lett. 2020;19(4):3101-10.

\section{Publisher's Note}

Springer Nature remains neutral with regard to jurisdictional claims in published maps and institutional affiliations.

Ready to submit your research? Choose BMC and benefit from:

- fast, convenient online submission

- thorough peer review by experienced researchers in your field

- rapid publication on acceptance

- support for research data, including large and complex data types

- gold Open Access which fosters wider collaboration and increased citations

- maximum visibility for your research: over $100 \mathrm{M}$ website views per year

At $\mathrm{BMC}$, research is always in progress.

Learn more biomedcentral.com/submissions 